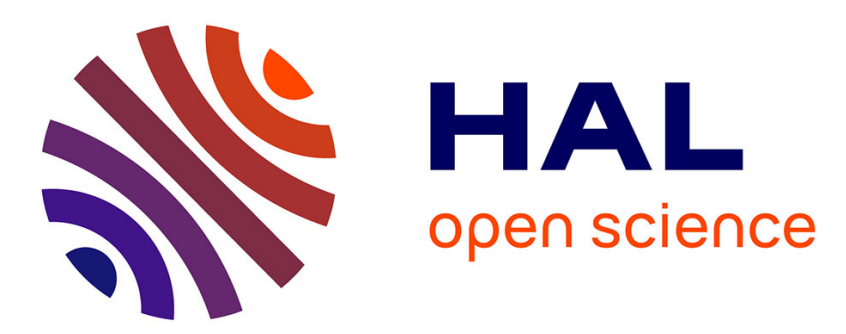

\title{
Observation d'une phase magnétique ordonnée à courte distance dans le spinelle normal $\mathrm{ZnCr2o4}$
}

\author{
R. Plumier, M. Lecomte, M. Sougi
}

\section{To cite this version:}

R. Plumier, M. Lecomte, M. Sougi. Observation d'une phase magnétique ordonnée à courte distance dans le spinelle normal ZnCr2o4. Journal de Physique Lettres, 1977, 38 (6), pp.149-151. 10.1051/jphyslet:01977003806014900 . jpa-00231347

\section{HAL Id: jpa-00231347 https://hal.science/jpa-00231347}

Submitted on 1 Jan 1977

HAL is a multi-disciplinary open access archive for the deposit and dissemination of scientific research documents, whether they are published or not. The documents may come from teaching and research institutions in France or abroad, or from public or private research centers.
L'archive ouverte pluridisciplinaire HAL, est destinée au dépôt et à la diffusion de documents scientifiques de niveau recherche, publiés ou non, émanant des établissements d'enseignement et de recherche français ou étrangers, des laboratoires publics ou privés. 


\title{
OBSERVATION D'UNE PHASE MAGNÉTIQUE ORDONNÉE A COURTE DISTANCE DANS LE SPINELLE NORMAL $\mathrm{ZnCr}_{2} \mathrm{O}_{4}$
}

\author{
R. PLUMIER, M. LECOMTE et M. SOUGI \\ Service de Physique du Solide et de Résonance Magnétique \\ C.E.N. Saclay, B.P. no 2, 91190 Gif sur Yvette, France
}

(Reçu le 13 janvier 1977, accepté le 9 février 1977)

\begin{abstract}
Résumé. - Des mesures de chaleur spécifique et de diffraction des neutrons indiquent qu'à $12,8 \mathrm{~K}$, $\mathrm{ZnCr}_{2} \mathrm{O}_{4}$ présente une transition du premier ordre entre un ordre magnétique à grande distance et un état d'ordre à courte distance.
\end{abstract}

Abstract. - Specific heat and neutron diffraction measurements indicate that at $12.8 \mathrm{~K}, \mathrm{ZnCr}_{2} \mathrm{O}_{4}$ undergoes a first transition from long-range magnetic order to short-range order.

En 1956, P. W. Anderson [1] note l'analogie existant entre la détermination de l'entropie de configuration de la glace à $0 \mathrm{~K}$ (problème historique résolu par L. Pauling [2] en 1935) et l'entropie de configuration de spins qui peuvent s'établir sur les sites octaédriques (sites B) d'un réseau spinelle. En l'absence d'autres interactions que les interactions antiferromagnétiques $J_{\mathrm{BB}}$ existant entre ions B premiers voisins, Anderson [1] montre qu'il existe un très grand nombre de configurations possédant toutes la même valeur propre $-2 J_{\mathrm{BB}}$ que celles des structures magnétiques ordonnées à grande distance [3]. Il suffit en effet pour cela que chacun des deux tétraèdres auxquels appartient un site $\mathbf{B}$ donné ait une polarité résultante nulle (Fig. 1 où $\bigcirc, \bigcirc, \oslash, \otimes$ correspondent aux

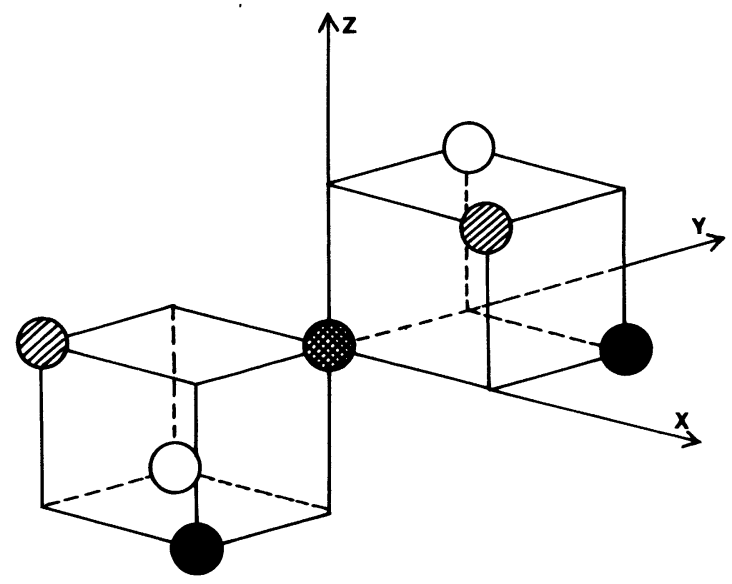

FIG. 1. - Site octaédrique et son environnement en premiers voisins.

[Octahedral site with first nearest neighbours.] directions positives ou négativẹ d'une ou deux directions d'antiferromagnétisme). Dans le cas d'une seule direction d'antiferromagnétisme et pour $N^{\prime}=2 N$ (par mole de ferrite $\mathrm{AB}_{2} \mathrm{O}_{4}$ ), le nombre de configurations $W_{N^{\prime}}^{\mathrm{I}}$ calculé par Anderson est alors [1] : $W_{N^{\prime}}^{\mathrm{I}}=2^{N^{\prime}}(3 / 8)^{N}$. De $S=k \log W$, on déduit alors $S^{\mathbf{I}}=0,808 \mathrm{cal}(\mathrm{mol} . \mathrm{deg})^{-1}$.

Des interactions magnétiques entre ions en sites $\mathrm{B}$ non premiers voisins existent cependant dans le réseau spinelle. Bien qu'elles soient d'un ordre de grandeur au moins inférieur à celui existant entre ions premiers voisins, elles sont toutefois responsables de la grande variété des structures magnétiques ordonnées à grande distance observées à basse température dans ces composés $[4,5]$. A plus haute température, dans l'expression de l'énergie libre, le terme $T S^{\mathbf{I}}$ peut, par contre, devenir supérieur à l'énergie des interactions magnétiques entre ions non premiers voisins. Seul subsiste alors un ordre à courte distance entre ions premiers voisins suffisant toutefois pour assurer la même valeur propre $-2 J_{\mathbf{B B}}$ au niveau de chaque site $B$. Il existerait donc une température de transition $T_{\mathrm{c}}$ telle qu'à des températures inférieures, on observerait l'ordre magnétique à grande distance alors qu'à des températures supérieures, un ordre magnétique à courte distance s'établirait sur les sites B.

Il peut paraître surprenant qu'aucune détermination de chaleur spécifique n'ait été effectuée dans le cas de tels composés au voisinage de leur température d'ordre alors que par diffraction des neutrons $[6,7]$, on observe non seulement que l'aimantation reste voisine de sa valeur à saturation jusqu'à la tempé- 
rature de disparition de l'ordre magnétique à grande distance, mais encore qu'au-dessus de cette température les réflexions magnétiques de Bragg font place à une bosse supposée d'origine magnétique.

Tel est le cas du spinelle normal $\mathrm{ZnCr}_{2} \mathrm{O}_{4}$ sur lequel des mesures calorimétriques viennent d'être effectuées dans notre laboratoire. La température de Néel de ce composé telle qu'elle se déduit du minimum de la courbe $\chi^{-1}(T)$ est de $13 \mathrm{~K}$. C'est aussi à cette température que, dans des expériences de diffraction des neutrons, les réflexions de Bragg d'origine magnétique font place à une bosse qui culmine à une valeur $\sin \theta / \lambda \simeq 0,12 \AA^{-1}$ (Fig. 2a). Comme l'aimantation qui se déduit des intensités des réflexions magnétiques de Bragg conserve pratiquement sa valeur à saturation jusqu'à $13 \mathrm{~K}$, la transition observée apparaît ainsi comme une transition du premier ordre.

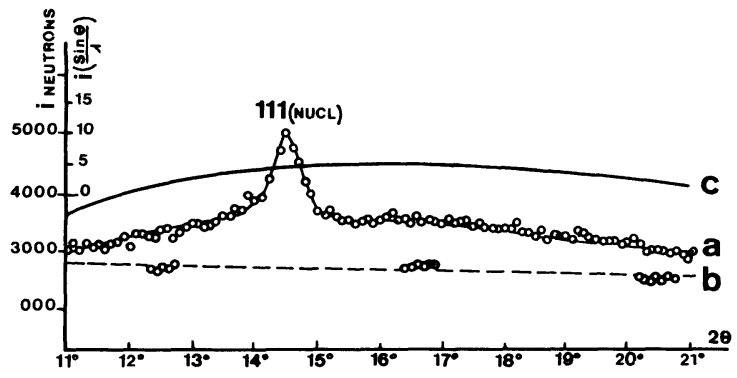

FIG. 2. - a) Spectre de diffraction des neutrons à $50 \mathrm{~K} ; b$ ) Fond continu à $4,2 \mathrm{~K} ; c)$ Fonction de Debye $I(\sin \theta / \lambda)$ corrigée du facteur de Lorentz.

(a) Neutron diffraction spectrum at $50 \mathrm{~K} ; b$ ) Background at $4.2 \mathrm{~K}$ c) Debye function $I(\sin \theta / \lambda)$ corrected for Lorentz factor.]

La chaleur latente de transformation que nous observons aux environs de $T_{\mathrm{c}}=13 \mathrm{~K}$ est de

$$
\Delta \theta=23 \mathrm{cal} . \mathrm{mol}^{-1} \quad \text { (Fig. 3) } .
$$

Cette valeur est d'un ordre de grandeur inférieur à l'énergie des interactions magnétiques entre ions $B$ premiers voisins, soit $480 \mathrm{cal}^{\mathrm{m}} \mathrm{mol}^{-1}$, cette dernière valeur pouvant se déduire, en bonne approximation, de la valeur observée de la température de Curie asymptotique $\theta=350 \mathrm{~K}$. Le $\Delta \theta$ observé est, par contre, de l'ordre de grandeur des interactions de super-superéchange existant entre ions seconds et troisièmes voisins $[4,5]$, c'est-à-dire des interactions assurant [1] à basse température l'ordre magnétique à grande distance.

De $\Delta \theta=23 \mathrm{cal} \cdot \mathrm{mol}^{-1}$, on déduit à la transition une différence d'entropie $\Delta S_{\mathrm{ex}}=1,8 \mathrm{cal}(\mathrm{mol} . \mathrm{deg})^{-1}$. Remarquons que cette valeur n'est que le $1 / 3$ de la différence d'entropie existant entre la phase paramagnétique et la phase magnétique ordonnée à saturation soit, par mole de ferrite

$$
\Delta S=2 R \log (2 S+1)=5,545 \mathrm{cal}\left(\mathrm{mol} \cdot \mathrm{deg}^{-1}\right) .
$$

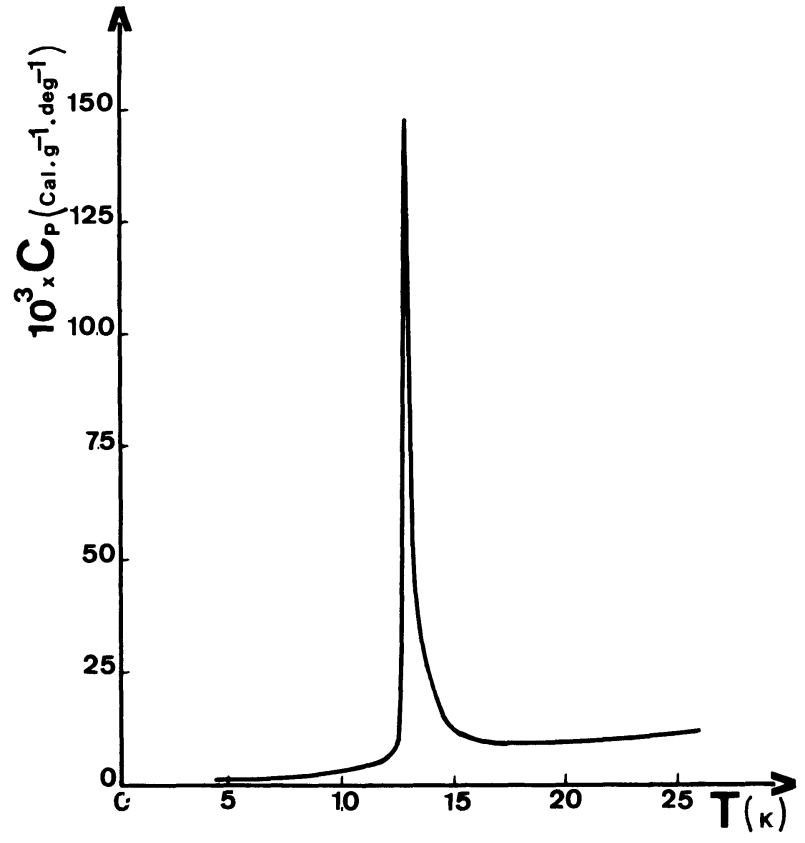

Fig. 3. - Chaleur spécifique à basse température.

[Specific heat at low temperatures.]

Cette valeur est, par contre, comparable à la valeur $S=0,808$ cal(mol.deg) ${ }^{-1}$ calculée par Anderson [1], l'accord pouvant d'ailleurs être amélioré si l'on remarque que la même valeur propre $-2 J_{\mathrm{BB}}$ est encore assurée au niveau de chaque site B si, au lieu d'une seule direction d'antiferromagnétisme, on en considère deux (Fig. 1). En effet, comme on le constate sur la figure 1 il est toujours possible de bâtir dans ces conditions des configurations de spins telles que, pour chacune d'elles, les deux tétraèdres auxquels appartient un site $\mathbf{B}$ donné aient une polarité résultante nulle. Le nombre $W_{N^{\prime}}^{\mathrm{II}}$ de ces configurations est alors $W_{N^{\prime}}^{\mathrm{II}}=4^{N^{\prime}}(9 / 64)^{N}$ d'où l'on déduit

$$
S^{\text {II }}=1,616 \mathrm{cal}(\mathrm{mol} \cdot \mathrm{deg})^{-1}
$$

soit une valeur d'entropie inférieure de $10 \%$ à celle observée expérimentalement.

Remarquons d'ailleurs que l'existence de deux directions d'antiferromagnétisme est suggérée par la structure magnétique ordonnée à $T<T_{\mathrm{c}}=12,8 \mathrm{~K}$. Le spectre de diffraction des neutrons de ce composé est en effet fort complexe $[6,7,8]$ et ne peut s'interpréter que par l'existence de deux directions d'antiferromagnétisme orthogonales.

Comme nous l'avons noté plus haut, la bosse d'origine magnétique observée par diffraction des neutrons à $T>12,8 \mathrm{~K}$ culmine à une valeur

$$
\left.1 / d \simeq 0,24 \AA^{-1} \quad \text { (Fig. } 2 a\right) \text {. }
$$

On remarquera que cette valeur correspond à la 
position du premier maximum de la fonction de Debye

$$
I\left(r_{i, j}\right)=f_{i} f_{j} \frac{\sin k r_{i j}}{k r_{i j}}
$$

pour une paire $(i, j)$ d'ions B premiers voisins couplés antiferromagnétiquement (Fig. 2c).

Ce résultat de diffraction des neutrons indique qu'à $T>T_{\mathrm{c}}$, les ions $\mathrm{B}$ premiers voisins restent couplés antiferromagnétiquement, alors que les interactions entre ions plus éloignés sont nulles en moyenne.

Il est intéressant de noter que dans l'étude par calorimétrie et diffraction de neutrons du thiospinelle $\mathrm{Cu}_{1 / 2} \operatorname{In}_{1 / 2} \mathrm{Cr}_{2} \mathrm{~S}_{4}$ effectuée récemment dans notre laboratoire [9], nous avons été conduits à une description de l'ordre à courte distance plus complexe que celle donnée ici puisqu'elle met en évidence le rôle joué dans cette gamme de températures par les interactions entre deuxièmes et troisièmes voisins. Un modèle semi-quantitatif de goutte magnétique intéressant 124 ions $\mathrm{B}$ a été donné dans ce cas [9] qui permet d'expliquer qu'à $T^{\prime} \simeq 5 T_{\mathrm{c}}$ où un autre pic de chaleur spécifique est observé, la phase magnétique à caractère liquide fait place à la phase paramagnétique.

Dans $\mathrm{ZnCr}_{2} \mathrm{O}_{4}$, l'ordre à courte distance n'intéresse que les six premiers voisins d'un ion $\mathbf{B}$ donné. Il n'est donc pas étonnant que la transition de disparition de l'ordre à courte distance ne puisse être mise en évidence par des mesures calorimétriques alors que des expériences de diffraction des neutrons indiquent que la bosse d'origine magnétique disparaît au-dessus de $100 \mathrm{~K}$.

\section{Bibliographie}

[1] Anderson, P. W., Phys. Rev. 102 (1956) 1008.

[2] Pauling, L., J. A. C. S. 57 (1935) 2680.

[3] Aiyama, Y., J. Phys. Soc. Japan 21 (1966) 1684.

[4] Baltzer, P. K. et al., Phys. Rev. 151 (1966) 367.

[5] Lotgering, F. K., J. Physique Colloq. 32 (1971) C1-34.

[6] Plumier, R., Sougi, M., C. R. Hebd. Séan. Acad. Sci. 268 (1964) 365 .

[7] Shaked, H., Hastings, J. M., Corliss, L. M., Phys. Rev. 1 (1970) 3116.

[8] Olés, A., J. Physique Colloq. 32 (1971) C1-328.

[9] Plumier, R., Sougi, M., Lecomte, M., à paraître dans Phys. Lett. 